\title{
Finite Element Analysis of Structural Dynamic Testing of Composite Quick-Install Scaffold
}

\author{
Ling CHEN ${ }^{a,{ }^{*}}$, Heng MA ${ }^{b}$, Yi-Li WEI ${ }^{c}$, Yi-Bo GAO ${ }^{d}, K_{\text {Ke }}$ QIAN $^{e}$ and Xie-Fei JIN ${ }^{f}$ \\ Zhejiang Huadian Equipment Testing Institute, Zhejiang Key Laboratory for Protection Technology \\ of High-Rise Operation, Hangzhou, Zhejiang, China ,310015 \\ a18458185160@163.com, 'bmahen38923@163.com, '63291550@qq.com, ${ }^{\mathrm{d}} 105569676 @ q q . c o m$, \\ eqianke10270@163.com, jjazhouyijie@163.com
}

Keywords: Composite quick-install scaffold, Modeling method, Finite element method, Impact test, Swing test.

\begin{abstract}
The general modeling method of composite quick-install scaffold used in substation construction is proposed for the scaffold inspection and evaluation in the delivery test. The method analyzes the characteristics of the scaffold structure and can be suitable for different types of composite quick-install scaffold. The strength analysis by finite element method is done for the HD-1 scaffold structure under the test conditions such as impact load and swing load. The stress variation of some key parts of the scaffold structure is shown for the test process, and the swing track of the whole structure is given in the swing test. These scaffold structure analyses can provide technical support for the performance evaluation to ensure the safety of electric power construction.
\end{abstract}

\section{Introduction}

Composite quick-install scaffold is the common apparatus used in ascending operation and maintenance work during the substation construction[1-2], shown in Fig. 1. The scaffold is mainly composed with glass fiber reinforced composites pipes, and its frame is connected by locking hook. The scaffold can be unarmed fast installed so it has good stability and the versatility.

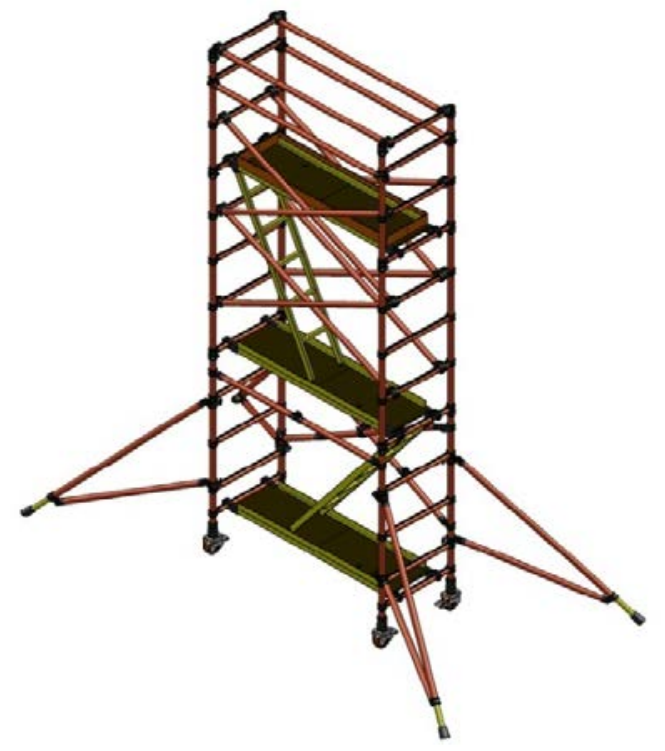

Fig. 1. HD-1 composite quick-install scaffold.

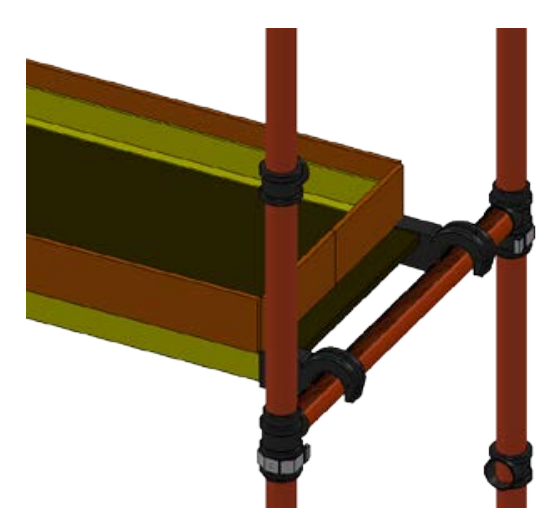

Fig. 2. The connection of platform and bar.

Composite quick-install scaffold must be carried out the type tests after completion of the design and production. The static test, horizontal dynamic impact test and recurrence swing test are included in the type test. Therefore the static and dynamic strength analyses of scaffold structure have very important position.

The scaffold is space truss structure, but the scaffold vertical bar and the horizon bar will be subjected to the transverse force (such as the bars in Fig. 2) and therefore the bar element cannot be 
used to analyze the scaffold structure in finite element[3]. And the strength of connection between the components of the scaffold structure is weak (such as the connection between platform and horizon bars in Fig. 2), so the connections cannot be set as fixed connections in finite element. Due to the above reasons, the computational complexity and workload of scaffold structure is greatly increased.

In this paper, a general modeling method of composite quick-install scaffold is proposed and the strength analysis of impact load and swing load test is done for the HD-1 scaffold by finite element method.

\section{Analysis of the Spatial Structure of Scaffold}

Scaffold is the complex spatial structure and its components can be divided into vertical bar, long horizon bar, short horizon bar, slant bar, leg and platform.

The different types of composite quick-install scaffold have the common characteristics, so the spatial position and the connection point of the components can be set on the basis of the dimension of the components and the deflection of the faces.

The space coordinates of other components can be obtained by the same way. The leg can be viewed as 2 independent bars, and the platform can be viewed as rigid body for its steel structure. Through the node matching, the information of connection points between the various components can be gotten.

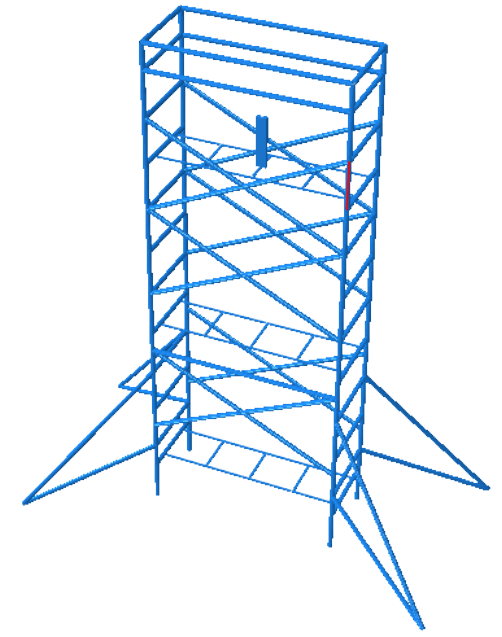

Fig. 3. Model of HD-1 Scaffold.

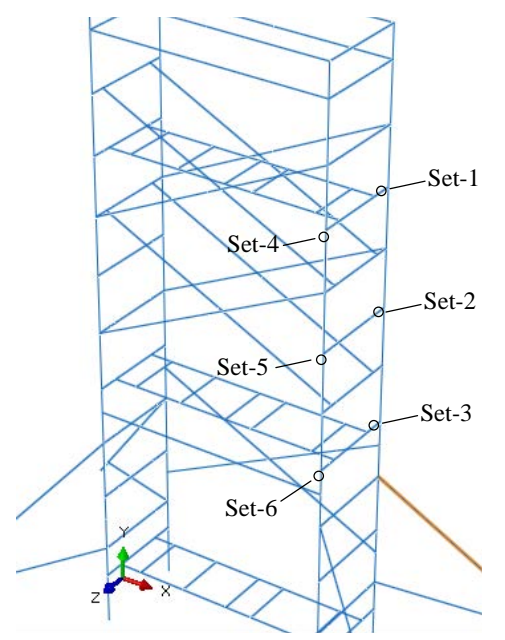

Fig. 4. The output sets.

According to the space coordinates, any type of scaffold structure model can be established. The model of HD-1 type scaffold was built as an example, shown in Fig. 3.

The foot of scaffold leg is supported on the ground without relative displacement, so the endpoint of leg is set with the fixed boundary condition. The foot of vertical bar is only restricted in the vertical direction displacement and the horizontal displacement is not restricted.

To analyze the dynamic characteristics of the structure, the Timoshenko beam element (B31) is used in the scaffold structure (the first-order element has concentrated mass, which is better than the second order element in simulation of stress wave effect)[4]. There are 879 elements(maximum length of element is $0.05 \mathrm{~m}$ ).

In order to analyze the stress change of scaffold in the process of unloading, there are 6 elements which can represent the max stresses of the structure to be selected as element sets to output data, shown in Fig. 4. 


\section{The Horizontal Dynamic Impact Test}

\section{Test Conditions}

According to the requirements of installation, there is 1.0 times the rated working load $(2 \mathrm{kN})$ at the top platform, and a horizontal load $(0.5 \mathrm{kN})$ at middle of the top long vertical bar along the width direction which will release after $1 \mathrm{~min}$ stable traction.

\section{Condition Setting}

The calculation result of the static analysis by FEM is introduced into the dynamic calculation as the initial state. The dynamic analysis process continues 2.2s.

Consider to the large energy dissipation caused by the local friction effect of scaffold components connection, the linear volume viscosity values for the damping is set as 2.5, and mass damping coefficient is 3.0, stiffness damping coefficient is 0 .

\section{Dynamic Analysis}

After unloading, the scaffold sloshing decreases due to the presence of damping, the overall kinetic energy is constantly decreasing. But the top vertical bar vibrates continuously during the dynamic process, so there is high frequency oscillation of the kinetic energy in decay process.

In the late decay elastic vibration disappears and the overall kinetic energy tends to be stable as shown in Fig. 5.

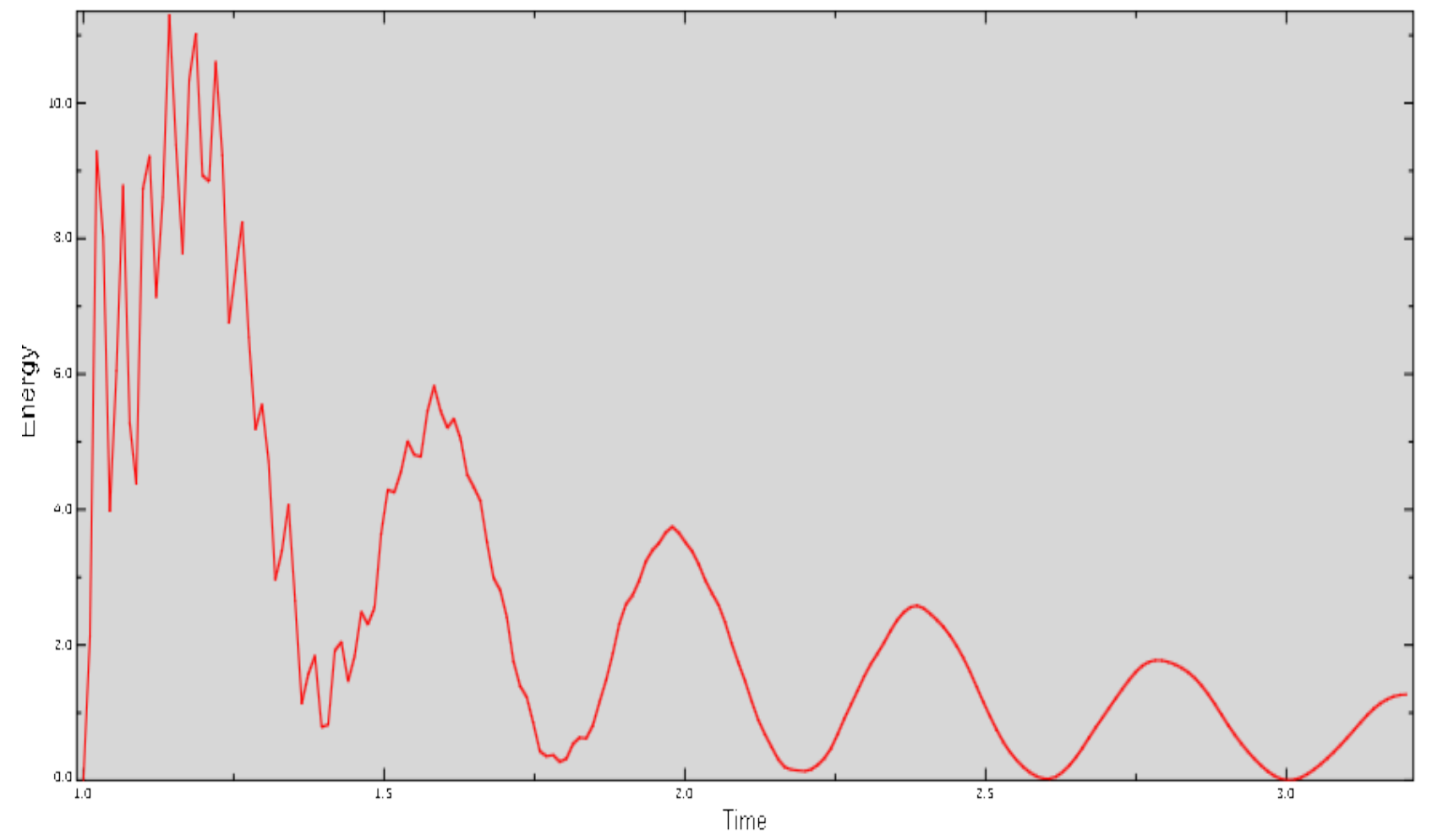

Fig. 5. Change of overall kinetic energy with time. 

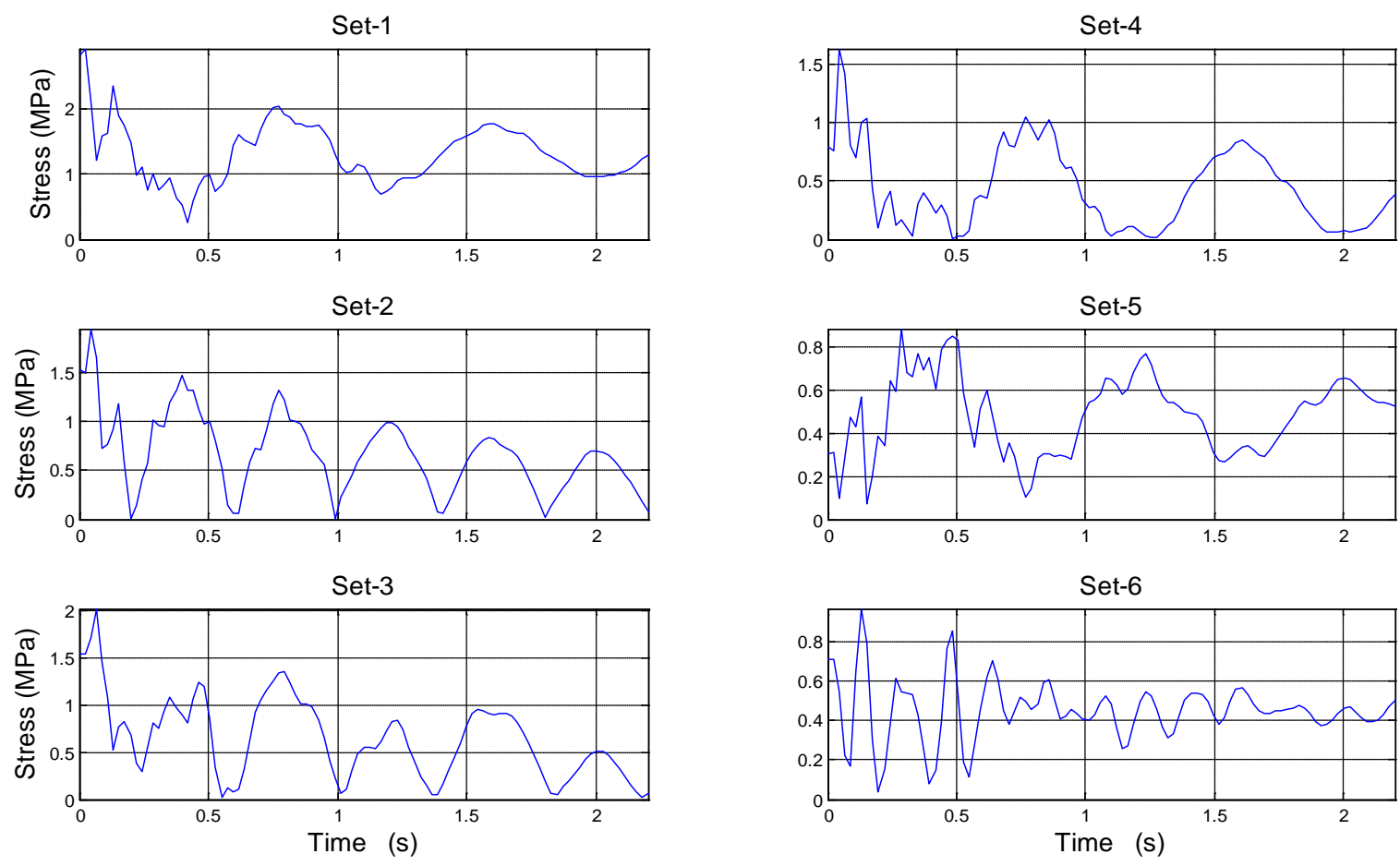

Fig. 6. The stress results of the element sets.

From the stress results shown in Fig6, the maximum Mises stress appears at Set- 1 in the unloading process of scaffold, which element bears the load of platform. The maximum result is only $2.64 \mathrm{MPa}$ which is far less than the composite yield strength, so the scaffold structure is overall safety.

\section{The Recurrence Swing Test}

\section{Test Conditions}

A swing fatigue test device( shown in Fig. 7) is placed on the center of top platform, and the swing block of the device moves back and forth 1000 times with amplitude $300 \mathrm{~mm}$ and the frequency $1 \mathrm{~Hz}$.

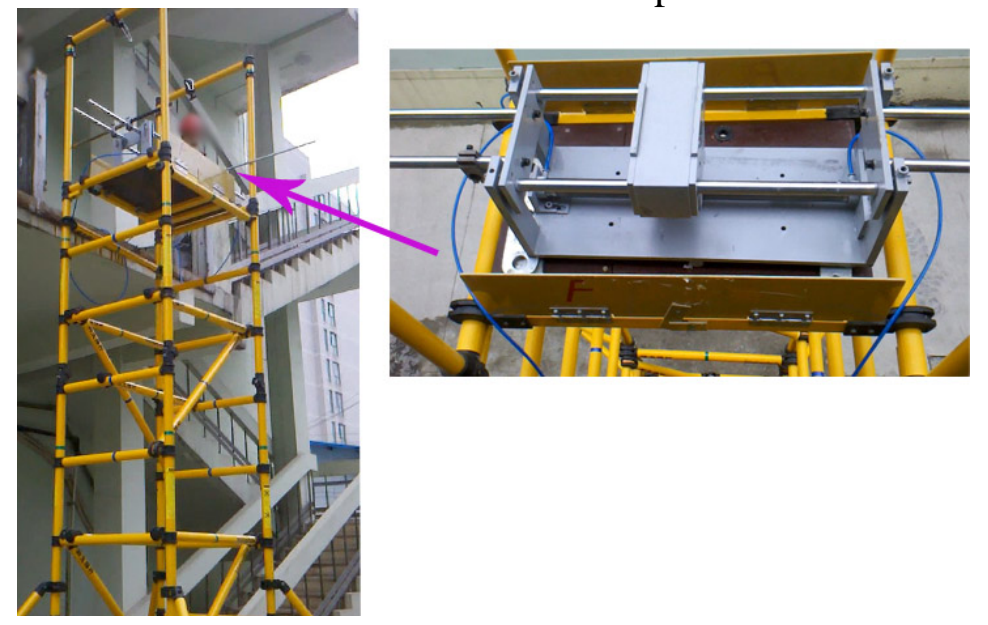

Fig. 7. The swing fatigue test device.

\section{Finite Element Analysis}

In the finite element model, the top platform is added to a 100kg mass (analog the swing block) which is connected with the platform and a $1.2 \mathrm{kN}$ periodic load on the block.

According to the previous calculations, the stress value of scaffold is far less than the yield strength so the scaffold can be considered as an elastic system and the frequency of external load is of 
the same order to the inherent frequency of scaffold. Therefore the transient model dynamic analysis is used for the instantaneous modal dynamic analysis.

\section{Frequency Analysis}

The structure frequency is needed for the transient model dynamic analysis. Due to many hinge connections of scaffold, there are lots of local vibration modes (only several parts deform) and these moving qualities is small to be neglected compared to the total scaffold quality. Only the first 8 order modes can meet the requirements which frequency is $1.61,1.63,2.07,5.51,5.69,6.30,7.35$, 7.98(Hz).

\section{Swinging Dynamic Analysis}

The load amplitude $A=1200 \mathrm{~N}$, frequency $\omega=1 \mathrm{~Hz}$, and the acting time is $4 \mathrm{~s}$.

According to the result, it can be seen that the structure will soon enter the stable response stage after the action of load, and the movement follows periodic motion with the same response frequency. The overall kinetic energy of structure is shown in Fig. 8.

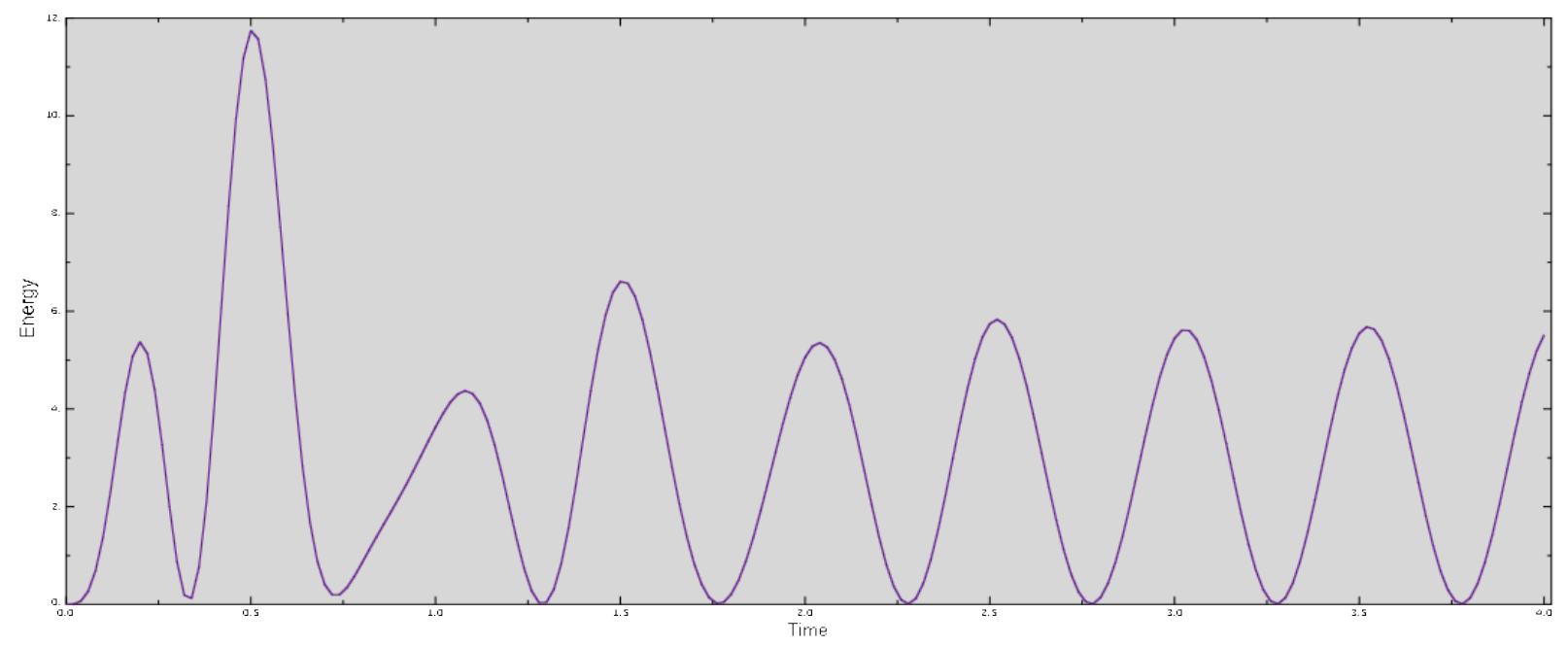

Fig. 8. Change of overall kinetic energy with time.

In order to show the vibration process more clearly, the 2 typical structural deformation of dynamic analysis are shown in Fig. 9.
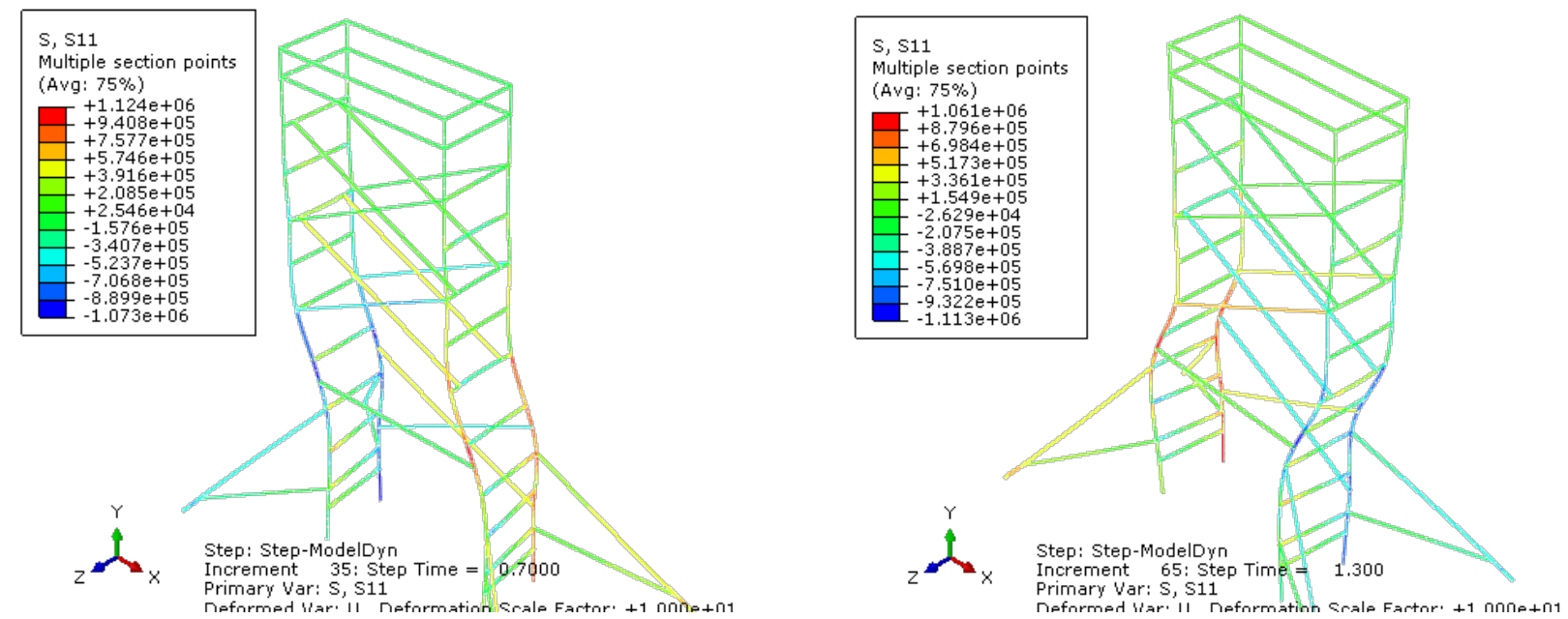

Fig. 9. The structural response deformation.

The scaffolding is a space frame structure, so there are periodic motions not only in $\mathrm{x}$ direction, but also in y direction during the swing test. The steady vibration response trajectory of a top-bar vertex on the $x-y$ plane becomes side-axis ellipse, shown in Fig.10. 


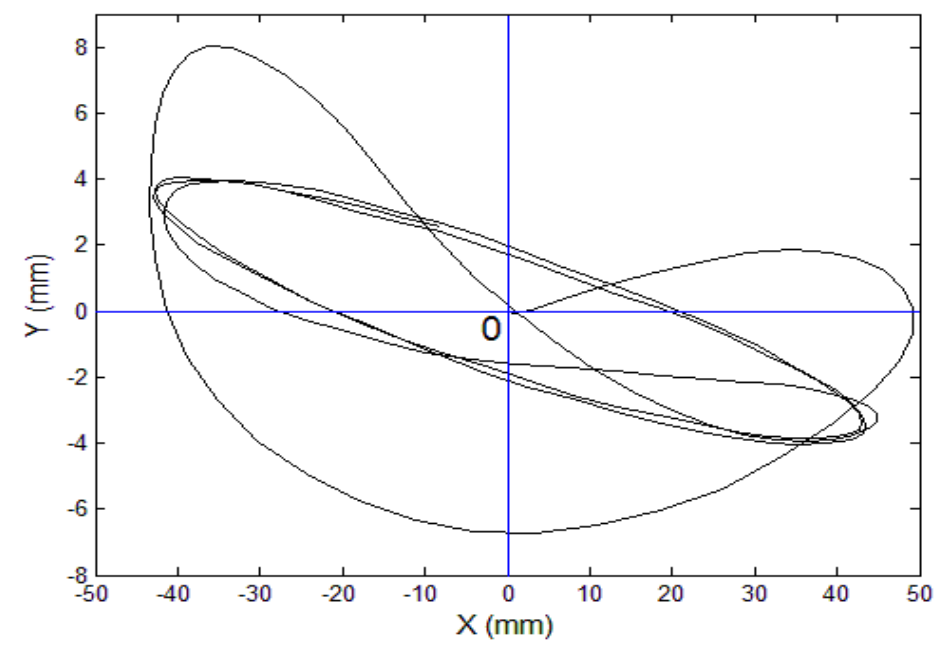

Fig. 10. The trajectory of top vertex on x-y plane.

The stress results of element sets are shown in Fig.11. The platform frame which has bigger stress value is made of steel, and stress is far less than the yield strength, so the analysis also does not take into account the platform frame.
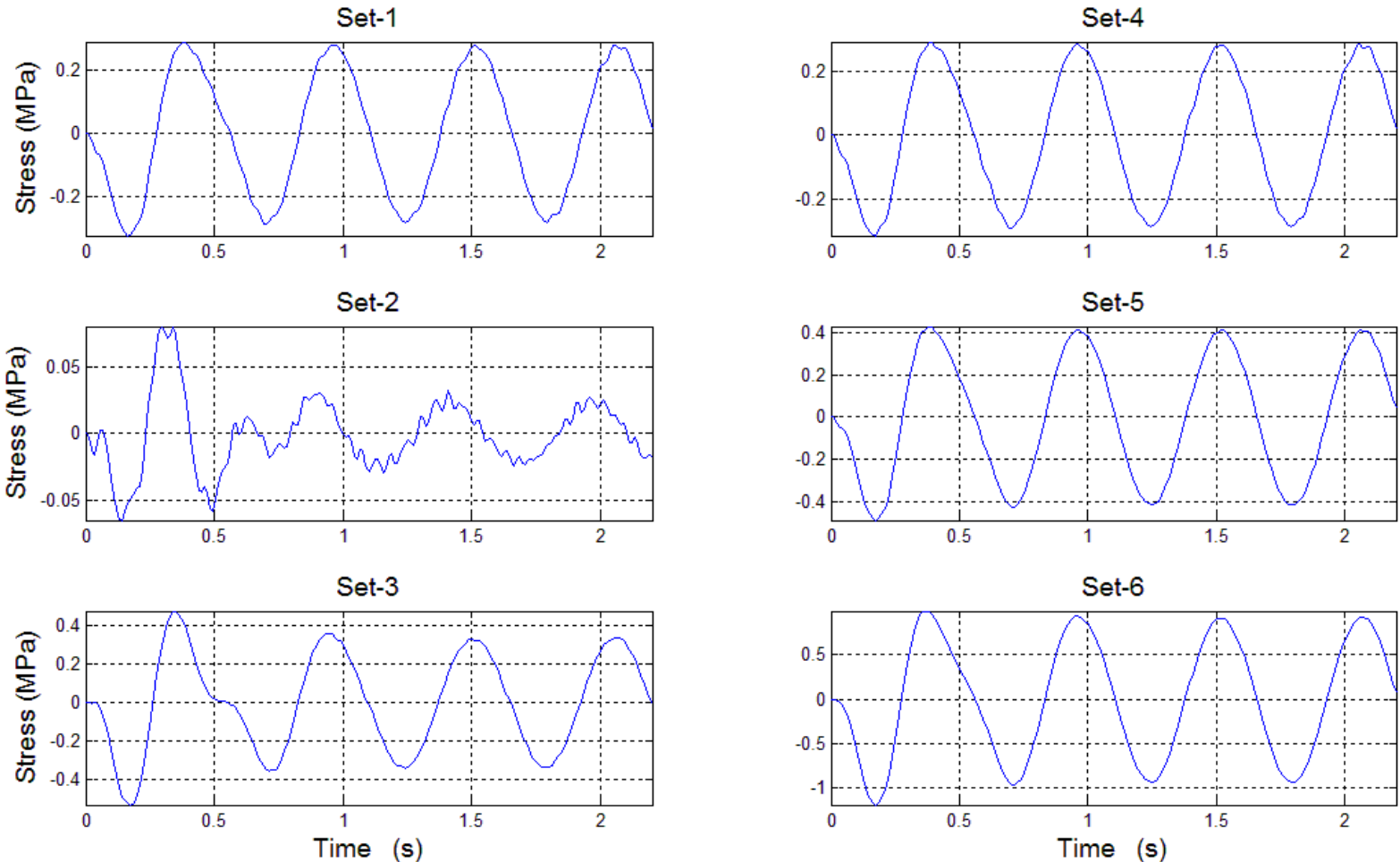

Fig. 11. The stress results of the element sets.

From the stress results, the stress frequency is same with the external load frequency. The maximum stress is in Set-6 which deformation is large and close to the scaffold leg, but stress value is only 1.0MPa far less than the yield strength of the composite. So the overall the scaffold structure is safety. The scaffolding is always in the elastic range, so it is not necessary to consider the effects of fatigue.

\section{Summary}

The finite element analysis of HD-1 composite quick-install scaffold structure is carried out based on the proposed scaffold design method, and the numerical simulation under horizontal dynamic impact 
load and recurrence swing load is done respectively for the deformation and stress state of scaffold structure. It can be seen that under the given load the most dangerous parts (maximum stress part) of scaffold structure is in the middle of the vertical bar. According to the calculation the maximum stress is much lower than the structural strength of the composite, so it is known the strength of composite quick-install scaffold meets the design requirements.

\section{References}

[1] Z.G.Ou, Q.H.Zhou, The Application of $10 \mathrm{kV}$ Fast-assembly/disassembly Live-line Work Scaffold. Guangdong Power Transmission Technology, 12(2015) 53-55. (in Chinese)

[2] H.Z.Tan, X.J.Zeng, F. Li. Study on the application method of highly insulating scaffold. DIANGONG WENZHAI, 4(2015) 65-67. (in Chinese)

[3] J.Z.Bai, Q.Sun, Y.N.Guo, Stress-analysis of a Complex Structure with the ANASYS System. Mechanical Science and Technology, 3(2003) 441-444.

[4] SIMULIA Co, ABAQUS Analysis User’s Manual, http://www.simulia.com 